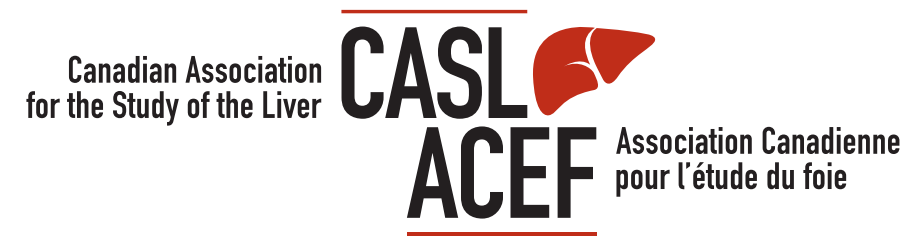

\title{
2012 Canadian Liver Foundation Operating Grants, Graduate Studentship and Summer Studentship Recipients
}

\begin{abstract}
A Chairs of the Research Committee, we are happy to announce the 2012 Canadian Liver Foundation (CLF) Operating Grants, Graduate Studentship and Summer Studentship Recipients. In addition, we thank all of the reviewers for their time and effort in selecting the recipients.

The CLF is the largest nonprofit funder of liver research in Canada and has invested more than $\$ 20$ million in research since it was first founded. The Foundation offers funding in three grant categories: Operating grants for senior and new researchers; Graduate studentships for $\mathrm{PhD}$ and Masters level researchers; and Summer Studentships for undergraduate students. The funds to support these research grants have been provided through donations from individuals and corporations across Canada, which have been made directly to the CLF to support liver research in Canada (these are not industry funded). Each of these awards is highly competitive and all grant submissions undergo a rigorous review process by the Research Committee, comprised of established liver researchers from around the world, who rate the submissions based on scientific merit and relevance to the CLF's priorities. For further information regarding these grants and how the support of CLF is an important contribution to the development and advancement of research on liver diseases, please visit the Canadian Liver Foundation website at www.liver.ca.
\end{abstract}

Christopher Rose PhD Claudia Zwingmann $\mathrm{PhD}$ CRCHUM, Université de Montréal, Montréal, Québec

\section{ANNOUNCING THE 2012 SUMMER STUDENTSHIP RECIPIENTS (\$4,000 FOR THREE MONTHS)}

Mr Andrew Collins, McMaster University, Hamilton, Ontario

Supervisor: Dr Gregory Steinberg

Project title: Treating non-alcoholic fatty liver disease by activating the AMP-activated protein kinase (AMPK).

Non-alcoholic fatty liver disease (NAFLD) is a common condition. The growing incidence of NAFLD is tightly linked to the obesity epidemic. NAFLD can also lead to other health problems including diabetes and heart disease, and affected individuals may require a liver transplant if inflammation and liver damage develop. The number of people who develop severe liver damage is likely to increase further as the rate of childhood obesity becomes more prevalent. Fat metabolism in the liver is being regulated by an enzyme that is switched on by exercise and drugs such as metformin and aspirin. Mr Collins will be testing whether metformin and aspirin might be effective in reversing NAFLD. The findings of this research will lead to new therapies for treating this very common liver disease, which currently has no cure.

\begin{abstract}
Ms Emma Torbicki, The Hospital for Sick Children, Toronto, Ontario

Supervisor: Dr Nicola Jones

Project title: Investigating the role of autophagy in ischemia reperfusion injury.

Currently, the short-term outcome after pediatric liver transplantation is excellent. However, the long-term outcomes for children who require liver transplantation need to be improved. During a transplant, when the liver is taken from the donor, the blood flow is cut off, causing lack of oxygen. Once the organ is placed in the patient and the blood vessels reconnected, the organ receives blood once again. This results in tissue damage due to the formation of toxins and invasion by white blood cells. Recent studies indicate that a specific cellular recycling pathway known as autophagy may help to remove the toxins and limit inflammation due to immune response. The results of this study will be the first step in determining whether autophagy is protective in this injury that occurs in transplanted livers.
\end{abstract}

\section{ANNOUNCING THE 2012 GRADUATE STUDENTSHIP}

RECIPIENTS (\$20,000/YEAR FOR TWO YEARS)

Ms Elizabeth Kuczynski, Sunnybrook Health Sciences Centre, Toronto, Ontario

Supervisor: Dr Robert Kerbel

Project title: Reversible resistance to sorafenib in hepatocellular carcinoma: Mechanisms and implications.

Worldwide, liver cancer is the third most common cause of cancerrelated deaths. Sorafenib is a drug that targets blood vessels that supply tumours with blood. Eventually, however, patients stop responding to drug treatment and develop resistance. Ms Kuczynski is studying how, contrary to common perception, resistance may be reversible and not permanent. By giving a prolonged time off a drug, cancer cells can be 'reset' and resensitized to once again respond to therapy. This research may have important implications for the clinical management of liver cancer and, potentially, other cancer-drug combinations in which resistance may be reversible.

\section{Mr Daniel Pang, University of Alberta, Edmonton, Alberta Supervisor: Dr Lorne Tyrrell}

Project title: Studying hepatitis A virus (HAV) infection, replication and clearance in a chimeric mouse model.

Hepatitis A virus (HAV) and hepatitis C virus (HCV) are two similar viruses that cause liver disease in humans. However, HAV primarily causes short-term disease while HCV usually causes long lasting disease for reasons that are not fully understood. While a better comparison of these two infections is needed, there is currently no small animal model to study HAV. Dr Tyrrell's laboratory has developed a mouse model with mixed human/mouse livers that are susceptible to HCV infections. The purpose of the study will be to test the model's susceptibility to HAV infection and to analyze its 
ability to fight off infections. Mr Pang will investigate whether the early immune response is sufficient to eliminate HAV in the mouse model. A better understanding of how we fight HAV and HCV infections may help in the development of a preventative vaccine for $\mathrm{HCV}$, one of the leading causes of liver cancer.

\section{Ms Mandana Rahbari, University of Alberta, Edmonton, Alberta \\ Supervisors: Drs Andre Mason and Michael Houghton}

Project title: Virological footprint of T-cell responses in patients with primary biliary cirrhosis.

Primary biliary cirrhosis (PBC) is known as a cholestatic liver disease because it blocks or stops the flow of bile. Although PBC is a disease of unknown cause, both genetic and environmental factors may influence the development of PBC. The objective of this research is to explain the relationship between an infection with the human betaretrovirus and $\mathrm{PBC}$ and determine whether infection with this virus plays a role in the development of $\mathrm{PBC}$. This knowledge could be used to design drugs to treat PBC.

\section{ANNOUNCING THE 2012 OPERATING GRANT RECIPIENTS (\$60,000/YEAR FOR TWO YEARS)}

\section{Dr Fernando Alvarez, CHU Sainte-Justine, Montreal, Quebec}

Project title: Depletion of $\mathrm{B}$ cells as a treatment for autoimmune hepatitis

Autoimmune hepatitis (AIH) is a liver disease caused by the body's own immune system, which attacks the liver resulting in inflammation and scarring. If left untreated, AIH is always fatal. AIH may present both as an aggressive form of acute hepatitis or a chronic illness that can progress to cirrhosis. Recently, Dr Alvarez and his team have discovered that a complete remission of $\mathrm{AIH}$ could be achieved in some patients with an antibody (rituximab) that temporarily destroys a type of white blood cells called B lymphocytes. However, AIH is believed to be caused by the attack on the liver by $\mathrm{T}$ lymphocytes and not by $\mathrm{B}$ lymphocytes. The goal of this research is to explain the mechanisms by which destruction of B cells help in the control of AIH. The research findings may lead to new treatments for $\mathrm{AIH}$, with fewer side effects than what are reported with current available therapy.

\section{Dr Klaus Gutfreund}

Co-Applicant: Dr Lorne Tyrrell, University of Alberta, Edmonton, Alberta

Project title: Identification and targeting of inhibitory T-cell signaling pathways in the duck hepatitis B model to explore novel immunotherapeutic strategies for chronic hepatitis B.

Chronic hepatitis B affects more than 350 million people worldwide and an estimated 300,000 people in Canada. Many patients with chronic hepatitis B will die due to cirrhosis and liver cancer. Current treatments with interferon-alpha and oral antiviral drugs effectively suppress the virus but most patients will not maintain a long-term response to therapy. Hence, a prolonged antiviral therapy is often required, which is costly, and many patients eventually develop drug resistance. Therefore, finding better treatments for hepatitis B remains a priority. The purpose of this research is to develop new treatment strategies that stimulate a successful immune response to resolve chronic hepatitis B infection. Dr Gutfreund's team has recently developed novel genetic vaccines and identified several molecules that can be targeted to improve antiviral immune responses. The focus of this research is to further explore these promising new therapeutic vaccines, which will lead to the development of new treatments for hepatitis B patients.

\section{Dr Mathieu Laplante, Laval University, Quebec City, Quebec}

Project title: Determination of the role of Deptor in the development of liver cancer.

Liver cancer is one of the most common cancers worldwide. Liver cancer is resistant to both conventional chemotherapy and radiation.
This leaves liver cancer patients with no effective therapeutic options and a very poor prognosis. Therefore, the development of more effective therapeutic tools to treat this disease is needed. A protein called mTOR is known to be commonly overactivated in cancer. This protein contributes to cancer growth by promoting protein and lipid synthesis. Recently, Dr Laplante has identified a new protein called Deptor that can inhibit mTOR. Deptor levels are low in liver cancer cells and preliminary experiments indicate that Deptor loss promotes tumour formation. The objectives of this research are to determine the role of this protein in liver cancer and to determine whether it could be targeted for the development of new treatment of liver cancer.

\section{Dr Andrew Mason, University of Alberta, Edmonton, Alberta}

Project title: Mouse models of genetic and environmental factors in primary biliary cirrhosis.

It is believed that primary biliary cirrhosis (PBC) results from an abnormal reaction of the body's immune system, possibly initiated by an infection. Designed to protect the body from infection, the immune system of $\mathrm{PBC}$ patients attacks the liver causing slow, progressive damage to the bile ducts. When the bile ducts are damaged, bile and other substances cannot be eliminated and accumulate in the liver. This eventually leads to cirrhosis. It is believed that both genetic and environmental factors may play a role in the development of PBC. Dr Mason has recently discovered that a virus found in PBC patients resembles a mouse virus known as mouse mammary tumour virus. A mouse model has recently been developed. This model has the same immune features that have been found in patients with PBC. Dr Mason has discovered that mouse mammary tumour virus is associated with the development of autoimmune biliary disease (similar to PBC) in these mice. Furthermore, Dr Mason has discovered that this biliary disease can be blocked by antiviral therapy. This research should lead to the development of treatments for patients with PBC.

\section{Dr Christopher Richardson \\ Co-applicant: Dr Eric Arts, Dalhousie University, Halifax, Nova Scotia}

Project title: Development of a novel hepatitis $\mathrm{C}$ virus cloning system in yeast to screen for infectious genomes and determine the role of neutralizing antibodies in viral clearance at acute infection.

Hepatitis C virus is responsible for 170 million infections worldwide and is a major cause of cirrhosis and liver cancer. However, $30 \%$ of those infected with the hepatitis $\mathrm{C}$ virus never develop chronic disease and get rid of the virus during the acute stage of the infection. Dr Richardson's research team proposes that the virus clearance is related to the rapid development of antibodies that neutralize the specific hepatitis C strains infecting the patients. This research requires the development of a unique yeast-based cloning system to produce infectious virus based on specific proteins derived from recently infected patients that either get rid of the infection or go on to develop chronic disease. Findings of this research will lead to the development of more effective treatments for chronic hepatitis C.

\section{Dr Naglaa Shoukry, Centre de Recherche du CHUM (Hôpital St-Luc), Montréal, Quebec}

Project title: How does the IL-28B polymorphism influence the outcome of acute hepatitis $\mathrm{C}$ ?

Chronic hepatitis $\mathrm{C}$ is a major cause of liver disease and liver cancer. We still do not understand how a small fraction of people exposed to the virus can get rid of it while the rest cannot. Recent studies have established a correlation between the variations in one gene known as IL-28B and the capacity to eliminate hepatitis C infection. However, how this gene variation influences the immune response against the virus is not yet known. Dr Shoukry believes that this gene controls the type of immune response that develops upon infection, in which patients who have the bad gene variant are not able to respond and, consequently, cannot clear the infection. The research findings will lead to the development of new treatments for hepatitis C. 


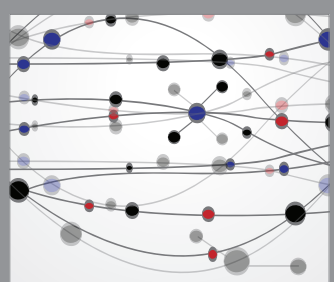

The Scientific World Journal
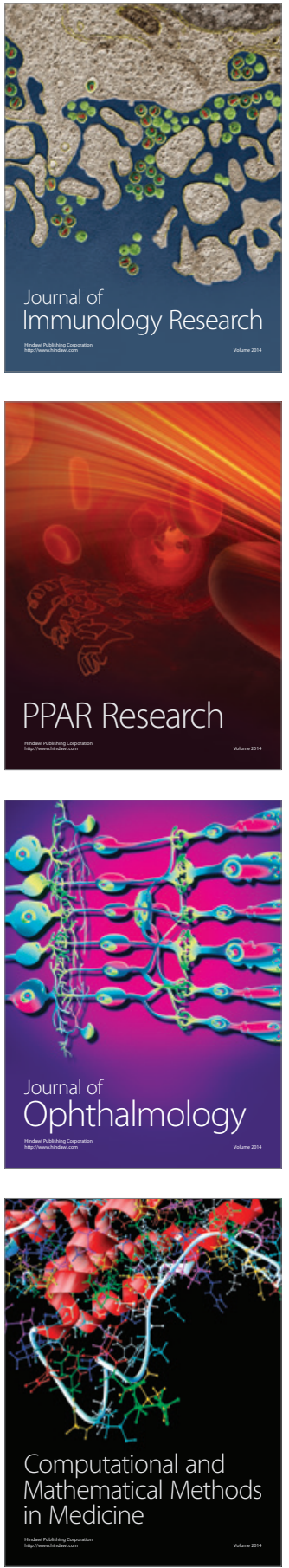

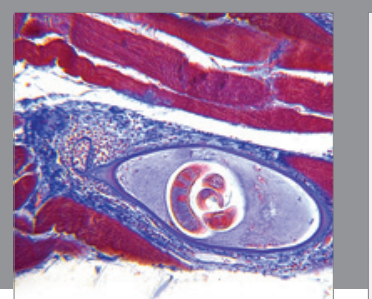

Gastroenterology Research and Practice

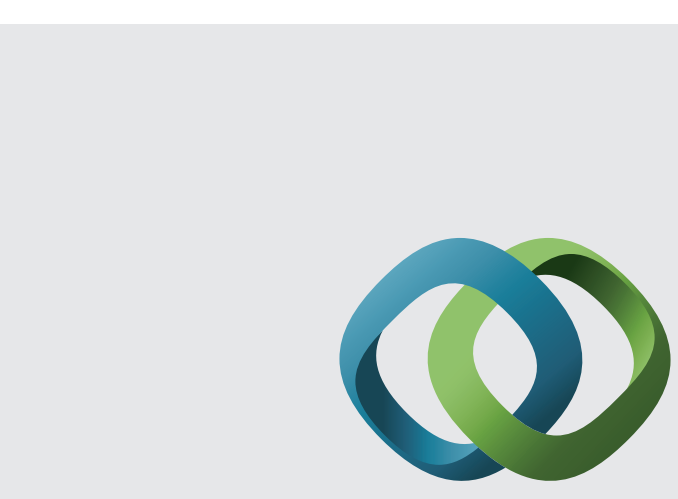

\section{Hindawi}

Submit your manuscripts at

http://www.hindawi.com
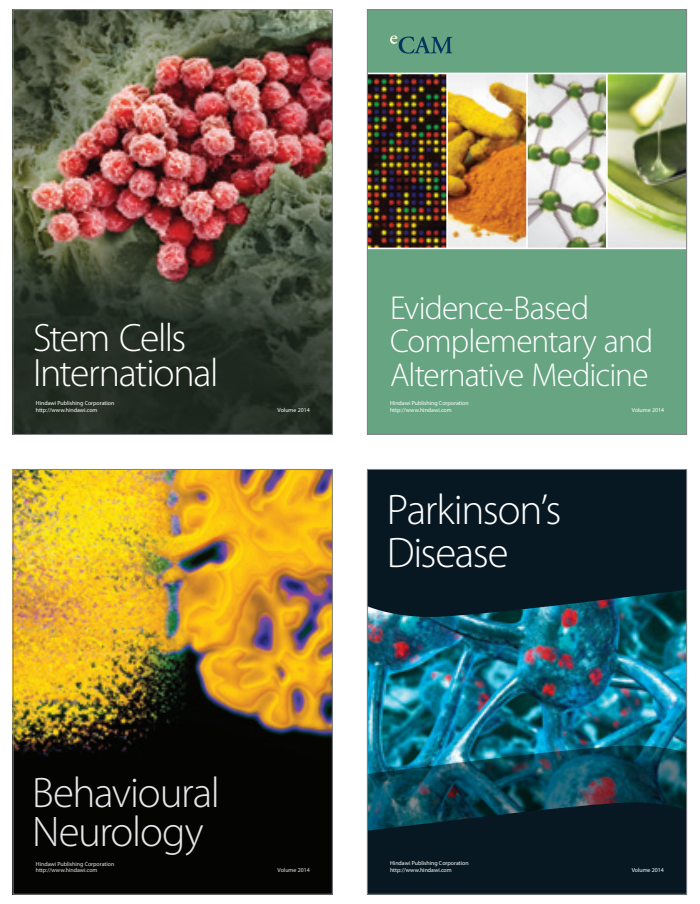
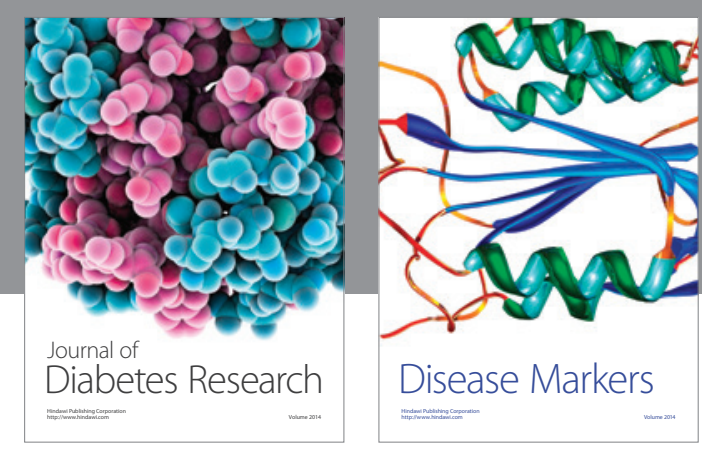

Disease Markers
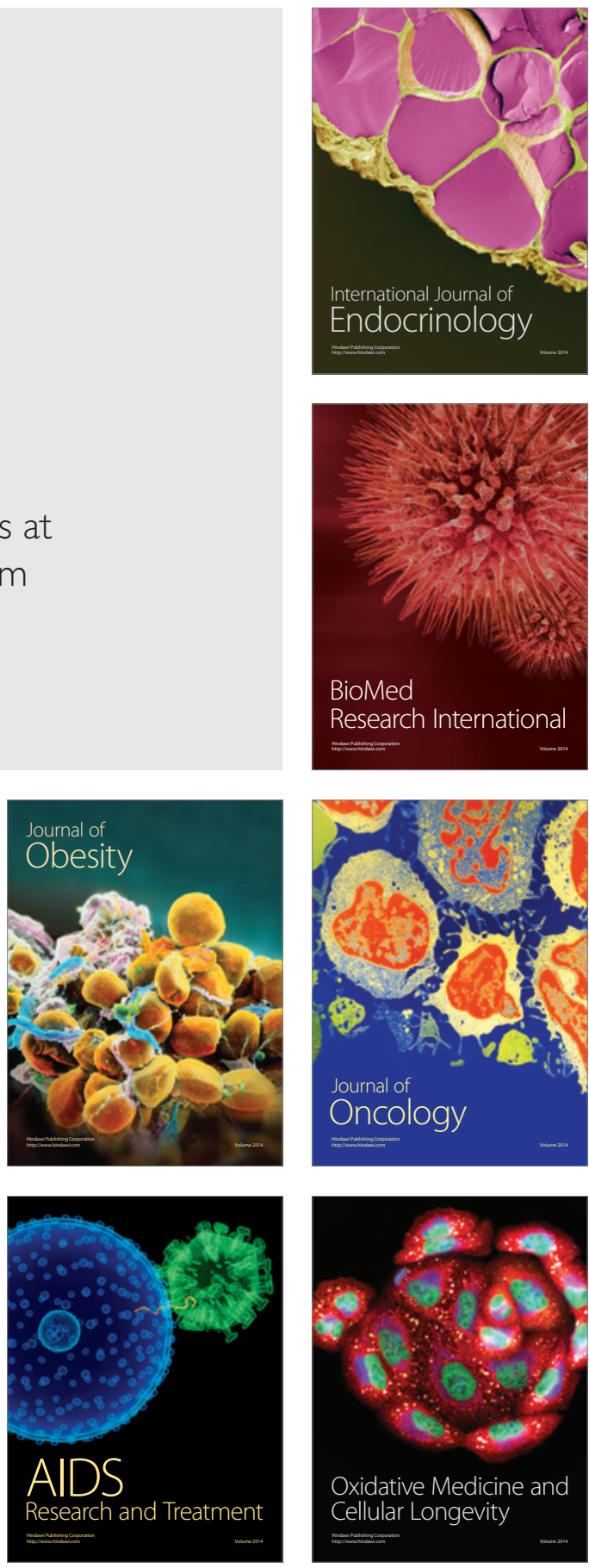